\title{
Forecasting droughts in East Africa
}

\author{
E. Mwangi ${ }^{1,2}$, F. Wetterhall ${ }^{2}$, E. Dutra ${ }^{2}$, F. Di Giuseppe ${ }^{2}$, and F. Pappenberger ${ }^{2,3}$ \\ ${ }^{1}$ Kenya Meteorological Department, Nairobi, Kenya \\ ${ }^{2}$ European Centre for Medium-Range Weather Forecasts, Reading, UK \\ ${ }^{3}$ College of Hydrology and Water Resources, Hohai University, Nanjing, China \\ Correspondence to: E. Mwangi (emwangi@meteo.go.ke)
}

Received: 24 July 2013 - Published in Hydrol. Earth Syst. Sci. Discuss.: 8 August 2013

Revised: 10 December 2013 - Accepted: 20 December 2013 - Published: 18 February 2014

\begin{abstract}
The humanitarian crises caused by the recent droughts (2008-2009 and 2010-2011) in East Africa have illustrated that the ability to make accurate drought forecasts with sufficient lead time is essential. The use of dynamical model precipitation forecasts in combination with drought indices, such as the Standardized Precipitation Index (SPI), can potentially lead to a better description of drought duration, magnitude and spatial extent. This study evaluates the use of the European Centre for Medium-Range Weather Forecasts (ECMWF) products in forecasting droughts in East Africa. ECMWF seasonal precipitation shows significant skill for March-May and October-December rain seasons when evaluated against measurements from the available in situ stations from East Africa. The forecast for OctoberDecember rain season has higher skill than for the MarchMay season. ECMWF forecasts add value to the consensus forecasts produced during the Greater Horn of Africa Climate Outlook Forum (GHACOF), which is the present operational product for precipitation forecast over East Africa. Complementing the original ECMWF precipitation forecasts with SPI provides additional information on the spatial extent and intensity of the drought event.
\end{abstract}

\section{Introduction}

Droughts have major economic and humanitarian impacts because rain-fed agriculture is the backbone of most economies in East Africa. The agricultural sector accounts both directly and indirectly for approximately 51,42 , and $25 \%$ of Kenya's, Uganda's and Tanzania's gross domestic product (GDP) respectively (Eguru, 2012). Over the last 5 decades, East Africa has experienced at least one major drought per decade (FAOSTAT, 2000), and there is a tendency of an increasing frequency and intensity of these events (AMCEN, 2011). Damage to the agricultural sector leaves the region exposed to the risk of famine, as demonstrated by the widespread famine and humanitarian crises caused by the two major droughts in the last decade (2008 2009 and 2010-2011). The ability to make accurate drought predictions with adequate lead time is therefore essential (Luo et al., 2008).

In a bid to ensure consistent access and interpretation of climate information, the World Meteorological Organization (WMO) initiated Regional Climate Outlook Forums (RCOFs) in various parts of the world. To coordinate action over the Greater Horn of Africa region, which the East African countries are part of, the Greater Horn of Africa Climate Outlook Forum (GHACOF, 2014) are held three times a year before the relevant rainy periods (March-May, JulyAugust, October-December). In preparation for each forum meteorologists from the National Meteorological and Hydrological Services (NMHSs) of Kenya, Uganda, Tanzania, Rwanda, Burundi, Ethiopia, Somalia, Djibouti, Eritrea, Sudan, and South Sudan (Fig. 1) convene at the Intergovernmental Authority on Development (IGAD) Climate Prediction and Applications Centre (ICPAC) to issue a joint forecast for the forthcoming season.

The forecast relies on a plethora of information. Firstly the analysis is performed on a sub-country level. Each country has been divided into homogeneous zones using principal component analysis (PCA) on a high-density historical precipitation data set. From this analysis only one representative rain gauge station was identified for each of the homogeneous zones. To issue a forecast for the next season, data from the representative stations are correlated with observed global sea surface temperature (SST) fields up to 6 months prior to the rainy season to identify ocean regions with a 


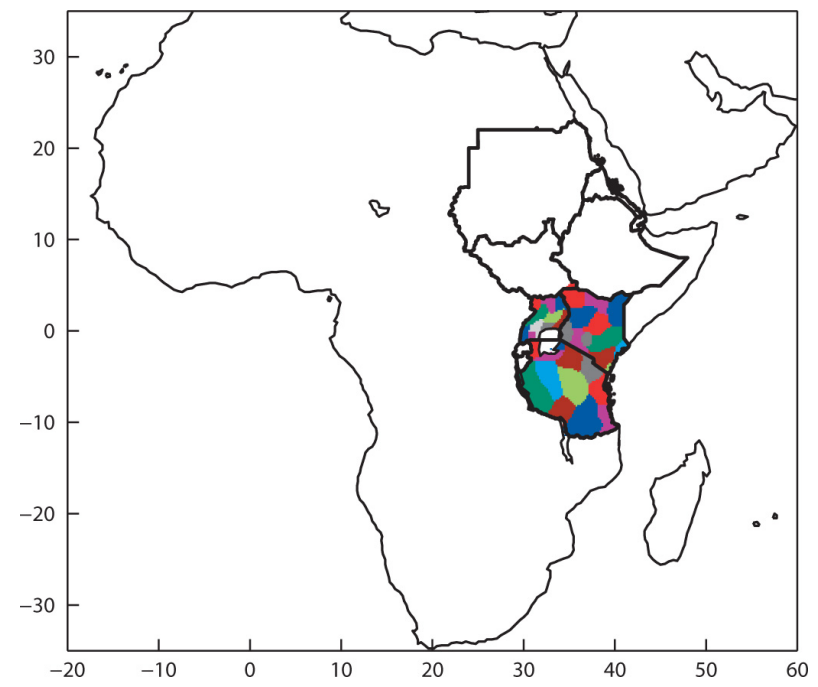

Fig. 1. Countries that participate in the Greater Horn of Africa Climate Outlook Forum (GHACOF; outlined) and homogenous zones over East African countries (coloured polygons) for which observations were available.

significant influence on the precipitation patterns (correlation values $>0.5$ ). SSTs from these ocean regions are used to develop forecast models using linear regression.

Afterward the forecast is upscaled to country level by comparing with forecasts made in neighbouring countries. If the forecasts do not agree, the forecasters look at historical years with similar SST patterns to select analogue situations for the target year. The forecasts are also compared with dynamical forecasts from several international centres. These centres were in 2006 assigned by the World Meteorological Organization (WMO) to deliver global seasonal forecasts as WMO Global Producing Centres (GPCs) for long-range forecasts. They have to release seasonal forecasts with global coverage and up to 4 months of lead time. The outcome is consolidated into what is known as the consensus forecast for the Greater Horn of Africa (Ogallo et al., 2008). The main product is a map showing the probability for the rainfall of the incoming season to be in one of the terciles - above normal, near normal or below normal - of the rainfall distribution as observed by the local rain gauge network. The consensus forecast is then used by the national meteorological services to disseminate press releases with advisories of expected floods (droughts) in zones with forecasts above (below) normal conditions.

The consensus forecast is an excellent forum to share observed data and local knowledge to coordinate naturalhazard-related political actions in the region. It nevertheless mostly relies on precipitation monitoring and past experiences to construct drought scenarios for the upcoming season. Unexpected weather conditions, such as extreme events outside the climatology, are not taken into account in this approach, and these conditions are likely to confound the forecaster's well-established knowledge. Moreover, the final product does not provide information on the spatial extent and intensity of droughts as it is mostly based on station data.

This study builds on the consensus forecast and explores the possible benefits of integrating the European Centre for Medium-Range Weather Forecasts (ECMWF) seasonal forecasting system product (SYS-4) into the procedure in place. SYS-4 is issued at the beginning of each calendar month and provides a 51-member ensemble forecast of precipitation up to 7 months ahead. If used in an automated system, it could extend the consensus drought forecast lead time and provide monthly updates in between the official forecasters' consensus meetings.

The paper is organised as follows: firstly the quality of the modelled precipitation is assessed through probabilistic skill scores in comparison with measurements from the available in situ network. Then an automated proxy of the consensus forecasts maps is created using the seasonal forecast. Finally, the capability of the seasonal forecast to complement the information already contained in the consensus maps is assessed at different lead times as a prototype of a reliable product for the future monitoring and forecast of drought in East Africa.

\section{Material and methods}

\subsection{Observations and forecast data}

The East Africa region comprises five countries: Kenya, Uganda, Rwanda, Burundi, and Tanzania. However, for this study rain gauge data only from Kenya, Uganda and Tanzania were available. This data set was used for the verification of SYS-4. A large part of East Africa experiences two distinct rainfall seasons: "long rains", which extend during March-May (MAM), and a season with "short rains", which lasts from October to December (OND). These seasons are linked to the movement of the Intertropical Convergence Zone (ITCZ) northward and southward (Nicholson, 1996). As a part of the consensus effort, the three countries have been subdivided into 34 homogeneous regions (see Fig. 1 for the consensus forecast boundaries) in terms of the precipitation climatology. Unfortunately there is no published literature to refer to how these regions were defined. Nonetheless the subdivision of these areas is a well-established practice used in GHACOF. Since this paper seeks to propose a methodology that could provide added information to the forecasters involved in the issuance of the consensus forecasts, it was decided to use the same subdivisions. Monthly rainfall totals for each of these 34 homogeneous zones were available for the period 1961-2009 through dedicated synoptic stations located in positions representative of each subregion. This data set provided both the climate information from which the consensus forecast anomalies were evaluated and the validation data for the drought forecasts. 
Past consensus outlooks and seasonal observation maps for the Greater Horn of Africa (GHA) region were sourced from the ICPAC as images. For a particular season, precipitation totals from representative stations from all the countries in the region are divided by the seasonal long-term mean (1961-1990) and to obtain the percentage of the longterm mean received in each particular season. The classes are selected as $<25 \%$ severely dry, $25-75 \%$ moderately dry, $75-125 \%$ normal, $125-175 \%$ moderately wet and $>175 \%$ severely wet. These values are at station points so, to produce the maps, interpolation is done.

The model used in this study was the ECMWF seasonal forecast system-4 (SYS-4; Molteni et al., 2011), which is a fully coupled system based on the Integrated Forecast System (IFS) cycle 36r4 atmospheric model version with TL255 corresponding to roughly $80 \mathrm{~km}$ spatial resolution and the Nucleus for European Modelling of the Ocean (NEMO; Madec, 2008) ocean model, which has a horizontal resolution of approximately 1 degree, and 42 levels in the vertical. On the first calendar day of each month, the system provides an ensemble of 51 simulations through initial condition perturbations derived from a combination of atmospheric singular vectors and an ensemble of ocean analysis. An extensive hindcast set of $30 \mathrm{yr}$ is also available for model calibration and verification. The set of hindcasts is initialised using ERA Interim reanalysis for the period 1981-2010 and has 15 ensemble members. Performances of the system to drive drought monitoring and forecasting in several African basins can be found in Dutra et al. (2013b).

\subsection{Quantitative assessment of the forecast skill}

The skill of SYS-4 precipitation forecasts was evaluated using standard scores applied to probabilistic forecasts; the scores are briefly presented in this section, and further details can be found in Appendix A. The scores include the anomaly correlation coefficient (ACC; Miyakoda et al., 1972) of the model and observations and the continuous ranked probability score (CRPS; Hersbach, 2000). The ACC provides information on the forecast skill of the ensemble mean (Hollingsworth et al., 1980; Simmons, 1986), whereas CRPS is a measure of the probabilistic skill of the forecast. The skill score that corresponds to the CRPS is the CRPSS. CRPSS values above (below) zero denote forecast skill better (worse) in comparison with reference forecast. The third score used was the area under the relative operating characteristic (ROC) curve that is based on ratios that measure the proportions of events and non-events for which warnings (a defined threshold) are forecasted (Mason and Graham, 2002).

SYS-4's predictive skill for precipitation over East Africa was assessed employing the hindcast data set for the period in which in situ measurements are available. Interpolation of the forecasts at station location was done using the nearestneighbour grid. Analogous analyses were performed using the average of four nearest points, and mean precipitation over the region (using the outlines in Fig. 1) providing very similar results (not shown).

\subsection{Qualitative assessment of the forecast skill}

Assessment of the added skill of using SYS-4 in the consensus framework was a more challenging task. Both the observed and the outlook maps were used as obtained from ICPAC since the original data set to reconstruct them was not available for this study. A quantitative assessment of the consensus maps was therefore virtually impossible. Instead, proxies of the consensus forecast maps were generated from SYS-4 forecasts for a subjective assessment on the basis of the added information they could potentially provide at a forecast meeting, such as the GHACOF. The exercise was repeated for the period of 2000-2010 and for both seasons, MAM and OND.

From the raw SYS-4 precipitation forecasts, dry and wet conditions were defined as the probability (or number of ensemble members) below the 30th percentile and above the 70th percentile of SYS-4's climatology for a particular season and lead time respectively. To condense this information into a single map for each lead time, the forecasts were binned into discrete categories as follows: moderately dry if $40 \%$ of the members predicted dry conditions, and the dry cases were more than wet cases; severely dry if $60 \%$ of the members predicted dry conditions; and extremely dry if $80 \%$ of the members predicted dry conditions. The same classification was applied for wet conditions, and the rest was classified as normal (or unclassified).

In addition to raw precipitation forecasts, maps of Standardized Precipitation Index (SPI) were calculated from hindcasts (> $30 \mathrm{yr}$ ) of SYS-4 precipitation. SPI is the index recommended by WMO for meteorological drought monitoring (WMO, 2009). Its calculation is based on long-term precipitation records, which are fitted to a cumulative probability distribution and then transformed into a standard normal distribution with mean zero for each month (Edwards and McKee, 1997). The gamma distribution was used in this paper for the first step in the transformation, and the calculation was done for each month separately. Positive (negative) SPI values indicate wet (dry) conditions. SPI can be calculated for any desired accumulation period, typically ranging from 1 to 48 months to reflect the impact of drought on the availability of the different water resources. Recently there has been increased focus on the use of SPI in drought forecasting. For example, Dutra et al. (2013b) proposed a methodology to forecast 3-month SPI for the prediction of meteorological drought over four basins in Africa based on the SYS4 forecasts of precipitation: the Blue Nile, Limpopo, Upper Niger, and Upper Zambezi. The detailed methodology used to calculate the SPI from the ECMWF seasonal forecasts is presented in Dutra et al. (2014). This followed the methodology initially described by Dutra et al. (2013b) and was 

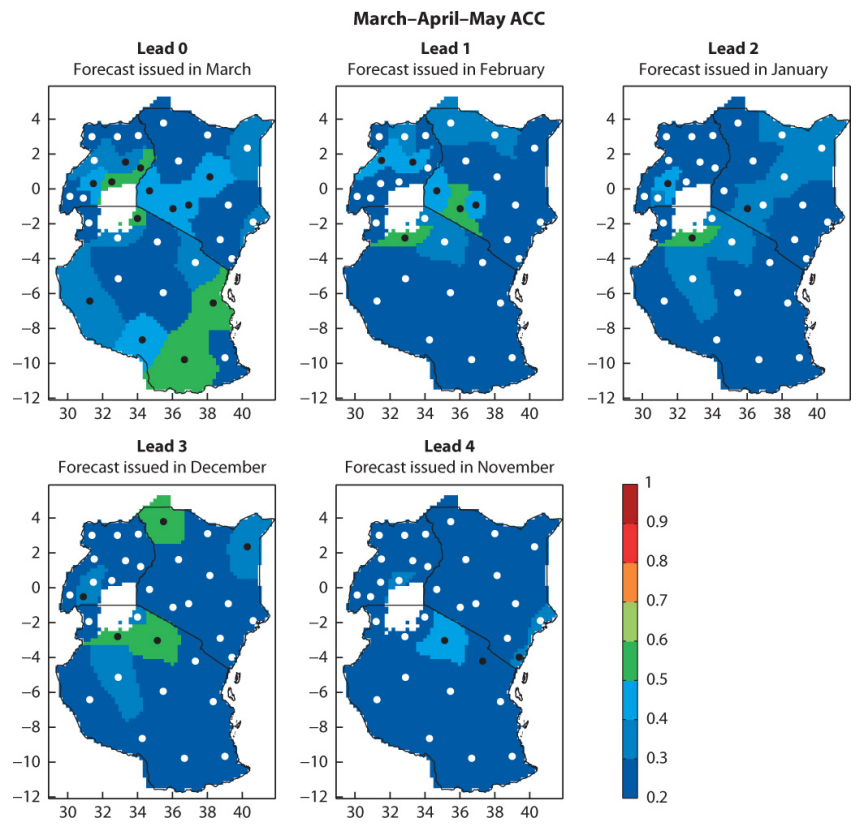

Fig. 2. Anomaly correlation coefficients (ACCs) between precipitation anomalies derived from ECMWF SYS-4 forecasts and in situ measurements during the MAM season for the period 19822009. Black and white dots represent regions where the correlation is statistically significant $(P<0.05)$ and insignificant $(P>0.05)$ respectively.

applied on a basin scale. Similar approaches have been also described by Mo et al. (2012) and Yuan and Wood (2013). The results display the ensemble mean of the SPI forecasts; the ensemble mean variance was rescaled for each lead time to conserve a standard deviation of 1 (see Dutra et al., 2014, for further details). While each ensemble member of the SPI forecasts follows the SPI characteristics of mean zero and standard deviation of 1 , the ensemble mean only conserves the zero mean and tends to have standard deviations lower than 1 . The rescaling of the ensemble mean variance is applied mainly to keep consistency of the SPI definition, in particular when presenting the results graphically.

\section{Results and discussion}

\subsection{SYS-4 verification against in situ observations}

As expected the predictive skill declines with increasing lead time (Figs. 2-4). The skill is higher in the OND than in MAM. Notable is that for both methods, there is higher skill in lead time 2 than lead time 1 for the OND season. This could be because of a negative drift of SYS-4's SSTs over the NINO 3.4 region (Molteni et al., 2011), which highly impacts precipitation over East Africa. The fastest drift of SSTs occurs during the boreal summer months. A bias in the near-equatorial winds in the west and central Pacific is the

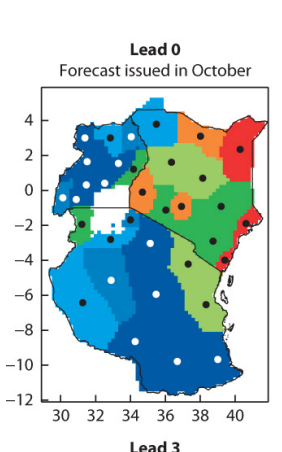

October-November-December ACC
Lead 1
Forecast issued in September
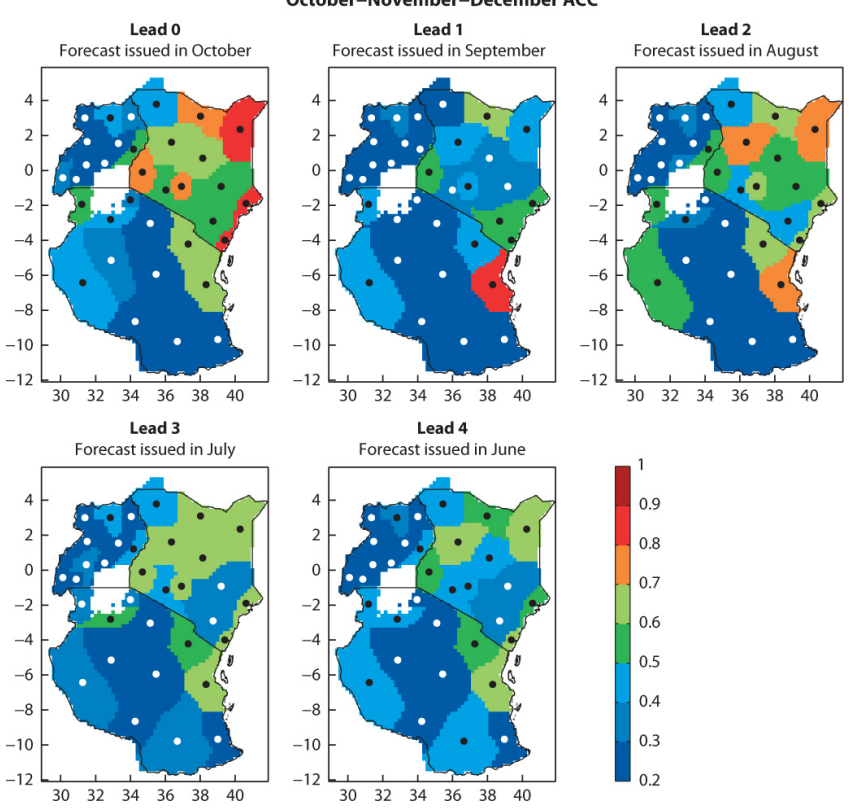

Fig. 3. As Fig. 2 but for the OND season.

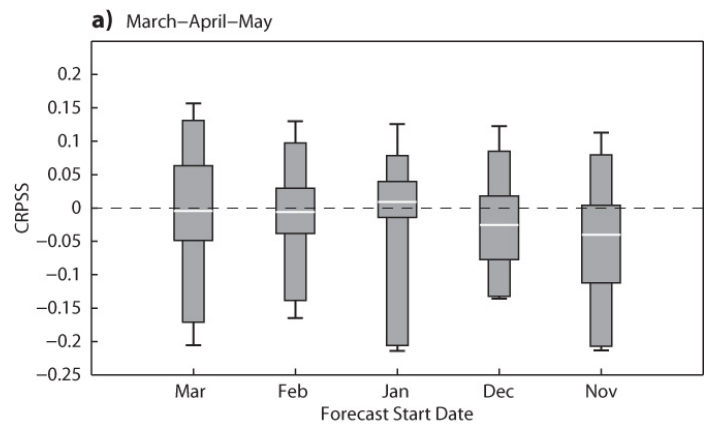

b) October-November-December

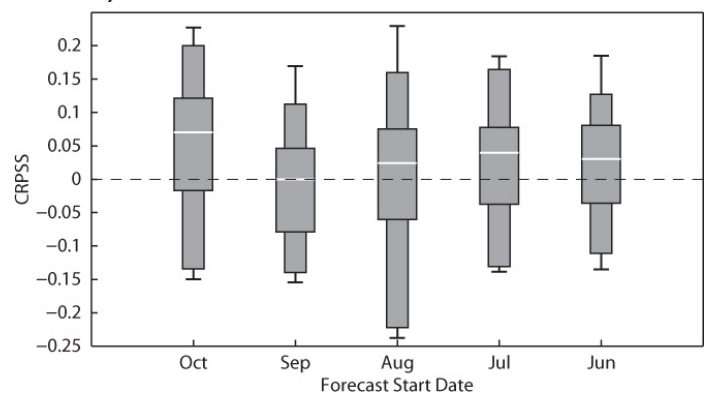

Fig. 4. Continuous ranked probability skill score (CRPSS) for MAM (a) and OND (b). The box plots extend from the minimum (whiskers), percentiles 10, 30, 50 (white line), 70, 90, and maximum.

dominant factor in driving an SST bias in the coupled model, whereby SSTs in the eastern equatorial Pacific drift to cold conditions (Molteni et al., 2011).

The ROC scores of the quantitative forecasts decline with increased lead time and there is higher skill for OND than 

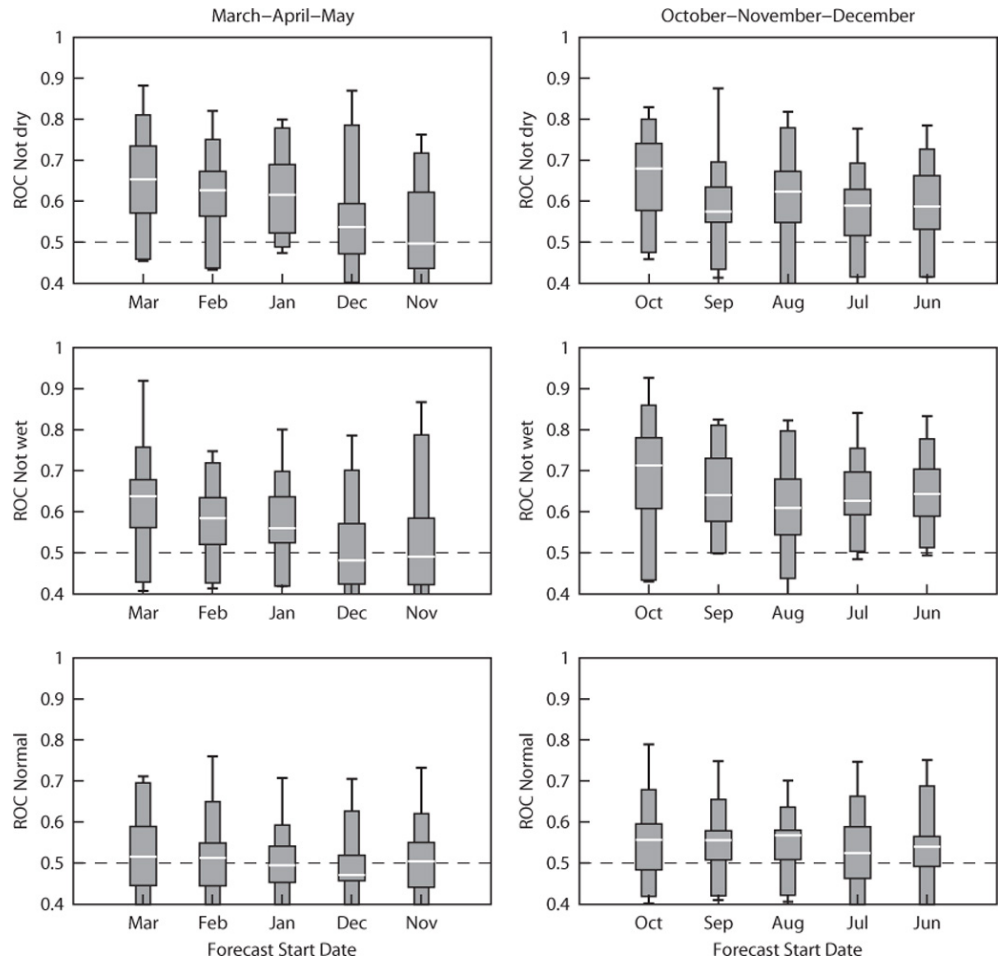

Fig. 5. Relative operating characteristic (ROC) scores for MAM (left panels) and OND (right panels). The box plots extend from the minimum (whiskers), percentiles 10, 30, 50 (white line), 70, 90, and maximum.
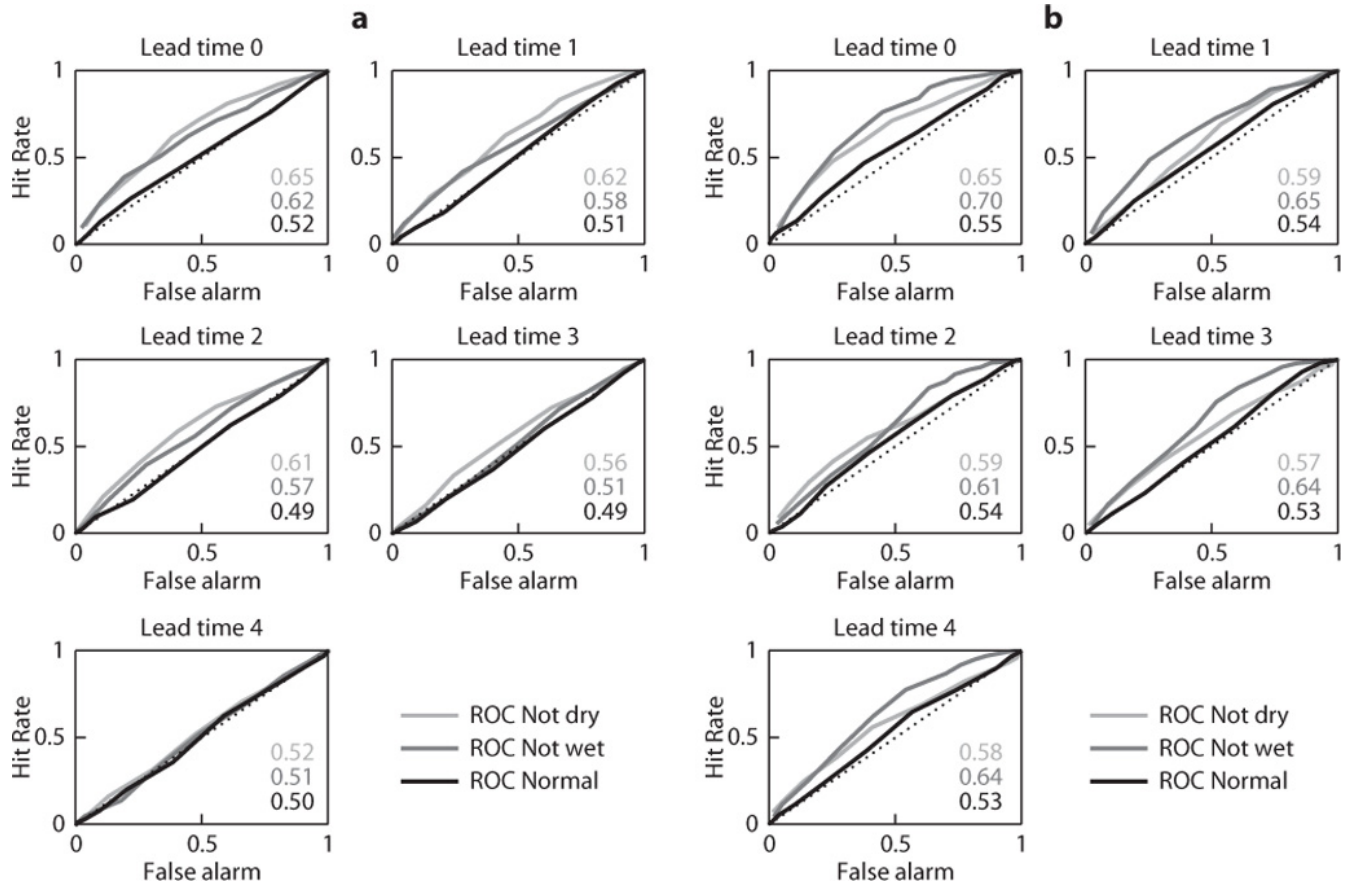

Fig. 6. ROC diagrams for MAM (a) and OND (b).

MAM, as in the previous results (Figs. 5 and 6). Over $50 \%$ of the stations have considerable skill for OND season for all lead times; this is the case from January for the MAM forecast (Fig. 5). SYS-4 has higher skill for the not dry (normal and wet) category in MAM for all lead times (Fig. 6). Since SYS-4 has a cold pool over equatorial Pacific, the seasonal forecasts always have a higher skill for La Niña conditions, which are associated with dry conditions over East Africa - 
thus the higher skill for not wet (normal and dry) category in the OND season (Fig. 6).

The high predictability in the Horn of Africa is well documented and is due to SYS-4's ability to capture the teleconnection between the Indian Ocean Dipole and ENSO. Generally, the predictive skill of SYS-4 is better in the OND season than the MAM season (Dutra et al., 2013a) due to the documented strong relationship between the OND seasonal rains and SST and ENSO (Mutai et al., 1998; Nicholson and Nyenzi, 1990; Ogallo et al., 1988). However, the MAM season rains have been associated with complex interactions between many regional- and large-scale mechanisms which generally induce large heterogeneities in the spatial rainfall distribution (Beltrando, 1990; Ogallo, 1982) and virtually negligible correlations with ENSO (Ogallo et al., 1988).

\subsection{Use of SYS-4 in the consensus framework}

Because of the subjective nature of the consensus forecasts, a purely quantitative assessment of its skill was not possible. We therefore resorted to performing a qualitative analysis based on subjective examinations of $11 \mathrm{yr}$ of forecasts. These analyses were performed independently by the five authors with the aim of judging the advantage SYS-4 would bring as an added product to the consensus framework.

Three cases were selected and discussed in detail to showcase the value SYS-4 could have added to the consensus outlook if it had been provided as precipitation probabilistic forecast and as SPI forecast. The three cases selected were seasons with below normal, normal and above normal precipitation.

In OND 2000, the observed precipitation was normal over most parts of the Greater Horn of Africa, except for some extremely wet patches over Ethiopia, Sudan, and Tanzania (Fig. 7). A significant area over north-eastern Kenya had moderately dry condition. SYS-4 precipitation forecast had a consistent signal for dry conditions over most of the region until August. September and October forecasts shift to normal conditions over the eastern part and wet conditions on the northern and western parts. Notable is that the two forecasts maintain a dry signal over northern Kenya and the Tanzania and Kenyan coast. When the same analysis was repeated with the SPI, a similar forecast evolution to the precipitation was observed but spatially smoother. The consensus outlook predicted climatological conditions for the northern part, wet conditions for the upper coastline and a small section of the western part, and normal conditions for the rest of the region. If SYS-4 September and October forecasts had been incorporated in the consensus forecast, then the outlook could have been adjusted for the Kenya coast, Ethiopia, and Sudan. That way the outlook would have been closer to the observations.

OND 2006 was a moderately wet season. From the observations most of the region experienced moderately wet conditions, and much of the coastal area experienced severely wet conditions (Fig. 8). The SYS-4 forecast had a wet signal far off in the ocean during June and August. The propagation of the wet signal inland happened in October. The same is seen in the SPI. However, the September forecast has a signal of moderately wet conditions inland. The consensus outlook forecasted normal conditions for most of the eastern part. If the consensus had been updated in October using SYS-4 forecast, the wet conditions observed on the eastern part could have been captured.

MAM 2009 was a moderately dry season for the eastern equatorial part of the region. Most of the northern parts experienced severely dry conditions, and normal conditions were experienced in the western part (Fig. 9). SYS-4 consistently captured the dry signal, but it only propagated inland in January and March for both precipitation and SPI. The consensus outlook predicted dry conditions over the eastern and a section of the northern part, and the western part had an above normal forecast. Combining the outlook and SYS4's March forecast would have helped adjust the wet forecast over Ethiopia and Sudan to dry conditions.

\section{Conclusions}

The Greater Horn of Africa Climate Outlook Forum takes place twice a year before the rainy seasons. During the event, by means of statistical downscaling and local knowledge, forecasters from the national meteorological centres of Kenya, Uganda, Tanzania, Rwanda, Burundi, Ethiopia, Somalia, Djibouti, Eritrea, Sudan, and South Sudan issue what is known as the consensus forecast for the Greater Horn of Africa. It consists of a map showing the probability for the rainfall of the incoming season to be in one of the terciles above normal, near normal or below normal - of the observed climatology.

In this work we have analysed whether or not the availability of long-range forecasts from ECMWF and the use of more specific drought indicators such as the SPI would bring added benefit to what is already in place. As a first step SYS4 precipitation forecasts were evaluated against station data over a vast part of the Great Horn of Africa using the historical data set which is also the reference for the consensus climatology. Considering the paucity of data in this area and the difficulty in obtaining a long-term data set, this by itself has represented a reality check of the performance for the system in a critical region for drought monitoring. SYS-4 has significant skill in forecasting precipitation over the study area with remarkably high skill in predicting the short rains (OctoberDecember) due to the strong predictability of the sea surface temperature in the Indian Ocean and the ENSO teleconnection (Hastenrath et al., 2004). The good performance of the system over the region is a good starting point; nevertheless the interest here is to understand if the availability of frequent updates from a dynamical model would add useful information to the already existing forecaster's interpretation of the statistical forecasts. While the subjective assessment 


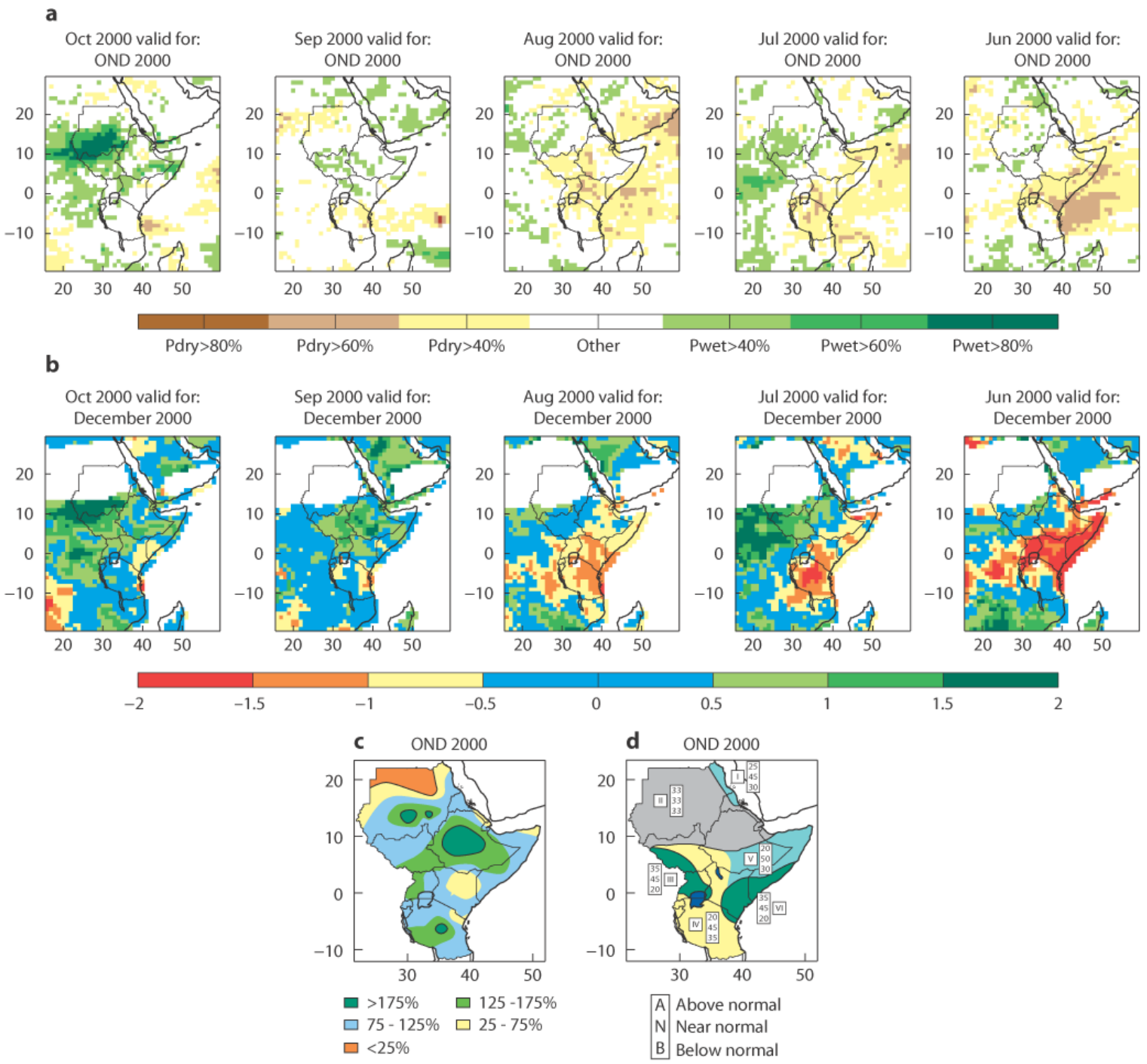

Fig. 7. SYS-4 probabilistic precipitation forecast for five lead times (a), SYS-4 3-month SPI forecast for five lead times (b), observed precipitation (c), and GHACOF consensus (d) all for OND 2000.

indicated that there would be an added advantage, no particular lead time stood out in provision of more information for the entire period, but in each season there was a lead time that would have made the consensus forecast better. The most interesting result is that if a drought index such as the SPI is used in place of raw precipitation data to generate a "proxy" of the consensus maps, then not only do the maps become spatially homogeneous as expected but also information about the intensity of the conditions expected in the coming season are made available. Such information could be used to support the decision process when issuing advisories for policy actions within the region.

\section{Appendix A}

\section{Verification scores}

The anomaly correlation coefficient (ACC) for a specific grid point or station is calculated as the Pearson correlation after removing the mean annual cycle of the observations $(Y)$ and forecasts ensemble mean $(\mathrm{X})$ :

$\mathrm{ACC}=\frac{\sum_{i=1}^{N}\left(Y_{i}^{\prime} X_{i}^{\prime}\right)}{\left[\sum_{i=1}^{N}\left(Y_{i}^{\prime 2}\right)\right]^{0.5}\left[\sum_{i=1}^{N}\left(X_{i}^{\prime 2}\right)\right]^{0.5}}$,

where $N$ is the number of forecasts, the subscript $i$ indicates the specific verification date, and the superscript ${ }^{\prime}$ denotes the temporal anomaly after removing the mean annual cycle. 


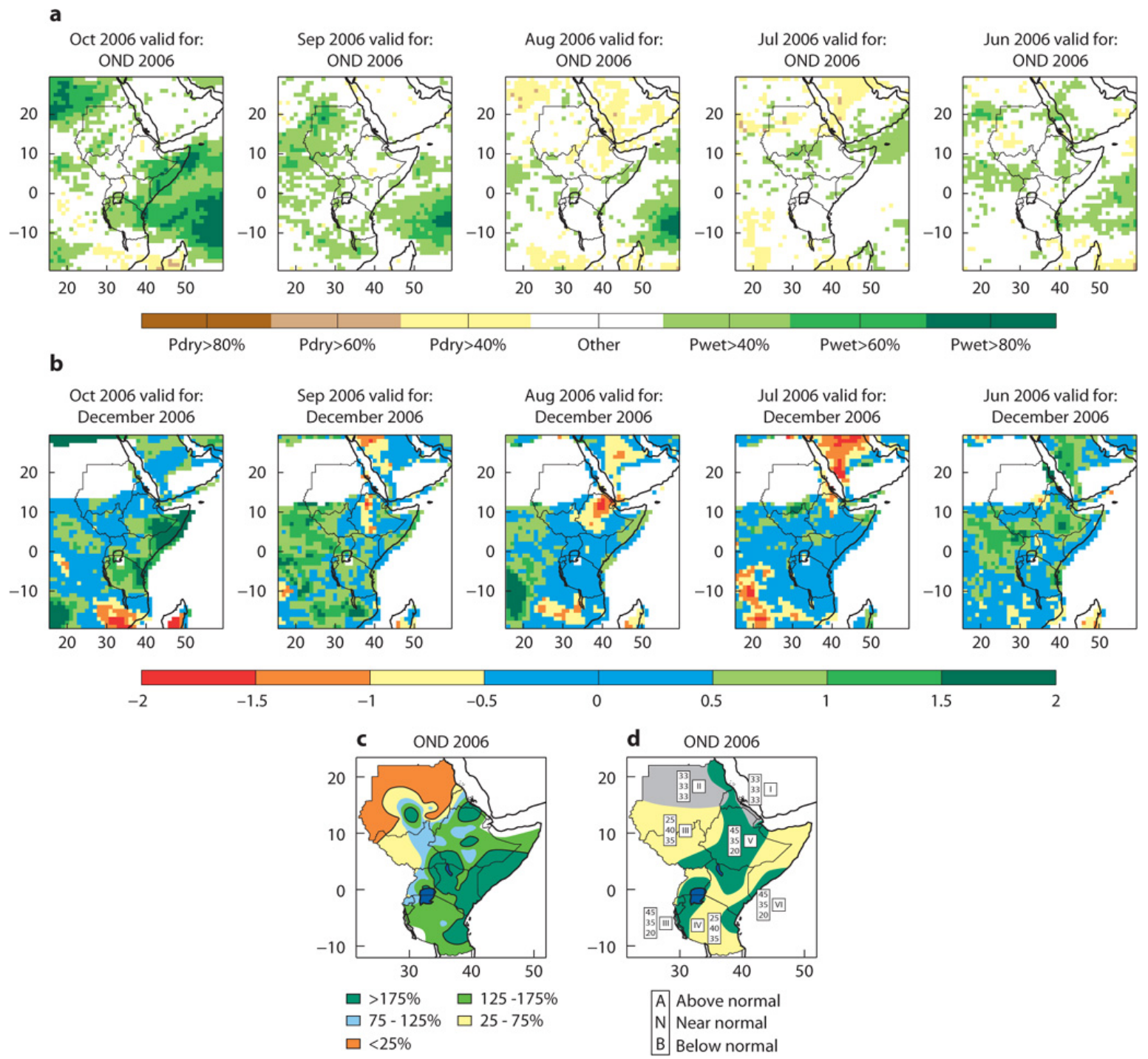

Fig. 8. SYS-4 probabilistic precipitation forecast for five lead times (a), SYS-4 3-month SPI forecast for five lead times (b), observed precipitation (c) and GHACOF consensus (d) all for OND 2006.

The continuous ranked probability scores (CRPSs; Hersbach, 2000) is a common tool to evaluate ensemble data:

$\operatorname{CRPS}=\frac{1}{N} \sum_{n=1}^{N} \int_{-\infty}^{\infty}\left[F(x)-H\left(x-x_{0}\right)^{2}\right] \mathrm{d} x$,

where $N$ is the number of forecasts, $F(x)$ the cumulative distribution function (c.d.f.) $F(x)=p(X \leq x)$ of the forecasts $x, x_{\mathrm{o}}$ the observations, and $H\left(x-x_{0}\right)$ the Heaviside function, which has the value 0 when $x-x_{0}<0$ and 1 otherwise. In order to quantify the skill of the probability score, the skill score is calculated as

$\mathrm{CRPSS}=1-\frac{\mathrm{CRPS}_{\mathrm{F}}}{\mathrm{CRPS}_{\mathrm{R}}}$ where CRPS $_{F}$ denotes the forecast score and $\mathrm{CRPS}_{\mathrm{R}}$ is the score of a reference forecast of the same predictand. The most commonly used reference forecasts are persistence and climatology. In this study, the reference forecast was a random sampling from previous years (climatology) with the same ensemble size as the forecasts. CRPSS values above (below) 0 denote forecasts that are better (worse) than the reference forecasts, and the maximum value is 1 .

The last score used in this study is the area under the relative operating characteristic (ROC), which is appropriate if an estimate of false alarm occurrence is important. The ROC is evaluated for a number of prescribed thresholds, where the probabilistic forecast is transformed into a binary number of each category defined by the thresholds. In a probabilistic forecasting system, there are various thresholds for each forecast category. For each of the thresholds, the correspondence 


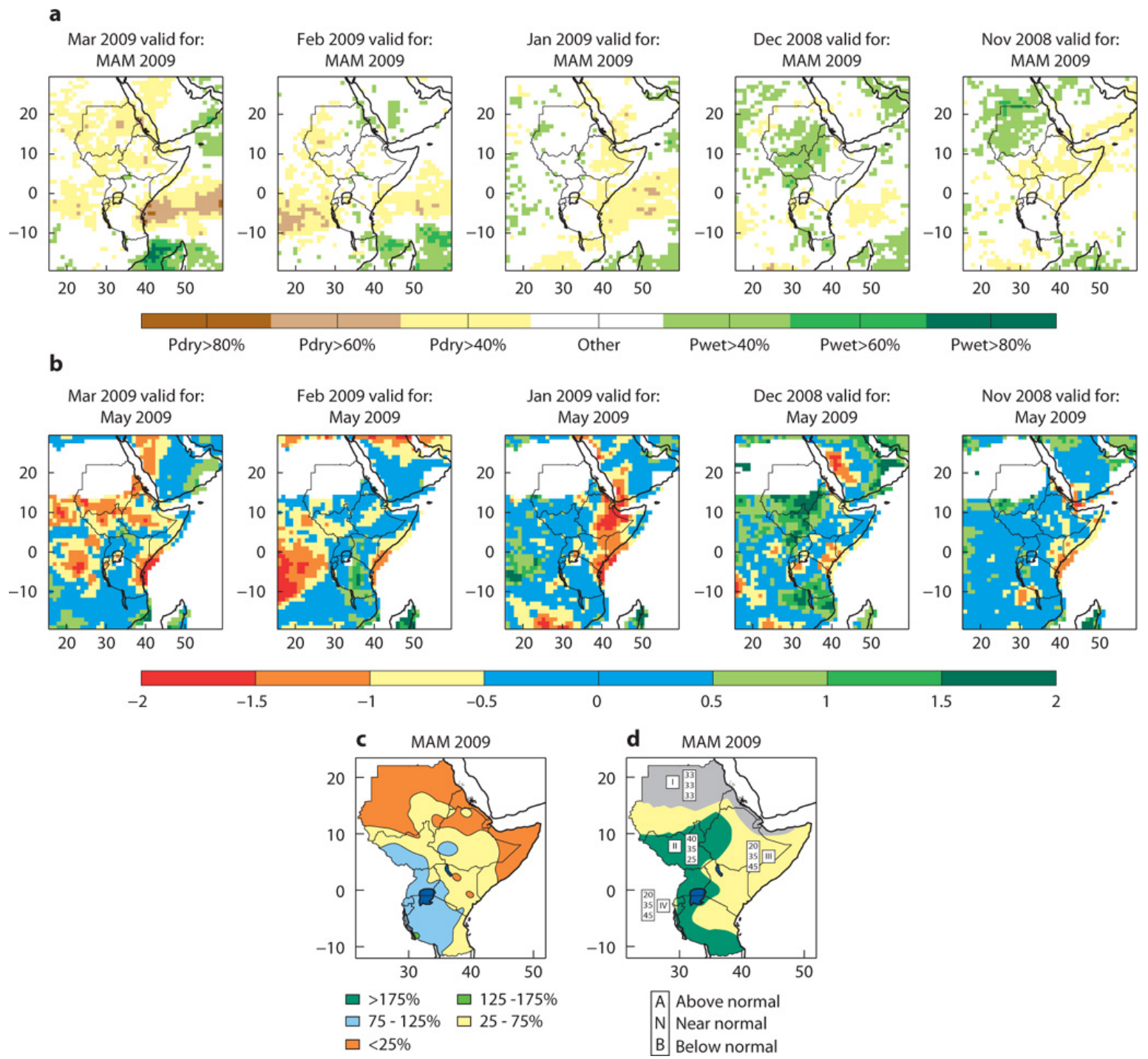

Fig. 9. SYS-4 probabilistic precipitation forecast for five lead times (a), SYS-4 3-month SPI forecast for five lead times (b), observed precipitation (c) and GHACOF consensus (d) all for MAM 2009.

between the forecasts (a sequence of dry or non-dry) and observations (a sequence of events or non-events) is evaluated. This results in a 2 by 2 matrix of the proportion of events for which a forecast was correctly issued (hit; $\mathrm{H}$ ), the proportion of non-events which were incorrectly forecasted (false alarm; FA), non-forecasted events (miss; M) and non-forecasted non-events (correct negative; $\mathrm{CN}$ ). The hit rate, defined as $\mathrm{H} /(\mathrm{H}+\mathrm{M})$, and false-alarm rate, defined as $\mathrm{FA} /(\mathrm{FA}+\mathrm{CN})$, give the ROC curve. Details on how to calculate ROC scores can be found in Mason and Graham (2002). ROC can vary between 0 to 1 (perfect forecast) with values below 0.5 denoting forecasts with no skill when compared with a climatological forecast.
Acknowledgements. We are grateful to Erland Källén, the director of Research at ECMWF, for supporting the visit of the lead author and to the Kenya Meteorological Department for supporting the same visit and affiliation at ECMWF. We are also grateful to ICPAC for providing the station data sets for East Africa and the consensus and observation maps for the Greater Horn of Africa region. Anabel Bowen is acknowledged for improving figure layout. This study was made available through a grant from WMO (4211-13/ETR/FKEN) and the European FP7 project DEWFORA grant no. 265454 .

Edited by: S. Maskey 


\section{References}

AMCEN (African Ministerial Council on the Environment): Addressing Climate Change Challenges in Africa, A Practical Guide Towards Sustainable Development, AMCEN, Nairobi, Kenya, 272 pp., available at: http://www.unep.org/roa/amcen/ docs/publications/guidebook_CLimateChange.pdf, last access: 3 February 2014, 2011.

Beltrando, G.: Space-time variability in April and OctoberNovember over East Africa during the period 1932-1983, Int. J. Climatol., 10, 691-702, 1990.

Dutra, E., Magnusson, L., Wetterhall, F., Cloke, H. L., Balsamo, G., Boussetta, S., and Pappenberger, F.: The 2010-2011 drought in the Horn of Africa in ECMWF reanalysis and seasonal forecast products, Int. J. Climatol., 33, 1720-1729, doi:10.1002/joc.3545, 2013a.

Dutra, E., Di Giuseppe, F., Wetterhall, F., and Pappenberger, F.: Seasonal forecasts of droughts in African basins using the Standardized Precipitation Index, Hydrol. Earth Syst. Sci., 17, 2359 2373, doi:10.5194/hess-17-2359-2013, 2013b.

Dutra, E., Pozzi, W., Wetterhall, F., Di Giuseppe, F., Magnusson, L., Naumann, G., Barbosa, P., Vogt, J., and Pappenberger, F.: Global meteorological drought - Part 2: Seasonal forecasts, Hydrol. Earth Syst. Sci. Discuss., 11, 919-944, doi:10.5194/hessd11-919-2014, 2014.

Edwards, D. C. and McKee, T. B.: Characteristics of 20th century drought in the United States at multiple time scales, Colorado State University, Fort Collins, Colorado, USA, Climatology Report Number 97-2, 174 pp., 1997.

Eguru, A.: Water Productivity in Agriculture: Challenges and Opportunities for Smallholder Farmers in the Drylands of Eastern and Southern Africa, Report, University of Nairobi, Kenya, 21 pp., 2012.

FAOSTAT: AQUASTAT Database, FAO, Rome, Italy, 2000.

GHACOF: available at: http://icpac.sbis.co.kr/, last access: 3 February 2014.

Hastenrath, S., Polzina, D., and Camberlin, P.: Exploring the Predictability of the "Short Rains" at the Coast of East Africa, Int. J. Climatol., 24, 1333-1343, 2004.

Hersbach, H.: Decomposition of the continuous ranked probability score for ensemble prediction systems, Weather Forecast., 15, 559-570, 2000.

Hollingsworth, A., Arpe, K., Tiedtke, M., Capaldo, M., and Savijärvi, H.: The performance of a medium-range forecast model in winter impact of physical parameterizations, Mon. Weather Rev., 108, 1736-1773, 1980.

Luo, L., Sheffield, J., and Wood, E.: Towards a Global Drought Monitoring and Forecasting Capability, in: 33rd NOAA Annual Climate Diagnostics and Prediction Workshop, 20-24 October 2008, Lincoln, NE, 2008.
Madec, G.: Nemo ocean engine, Note du Pôle de modélisation 27, Institut Pierre-Simon Laplace(IPSL), Paris, France, 209 pp., 2008.

Mason, S. J. and Graham, N. E.: Areas beneath relative operating characteristics (ROC) and relative operating levels (ROL) curves: Statistical significance and interpretation, Q. J. R. Meteorol. Soc., 128, 2145-2166, 2002.

Miyakoda, K., Hembree, G. D., Strickler, R. F., and Shulman, I.: Cumulative results of extended forecast experiments, I. Model performance for winter cases, Mon. Weather Rev., 100, 836-855, 1972.

Mo, K. C., Shukla, S., Lettenmaier, D. P., and Chen, L.-C.: Do Climate Forecast System (CFSv2) forecasts improve seasonal soil moisture prediction?, Geophys. Res. Lett., 39, L23703, doi:10.1029/2012g1053598, 2012.

Molteni, F., Stockdale, T., Balmaseda, M., Balsamo, G., Buizza, R., Ferranti, L., Magnusson, L., Mogensen, K., Palmer, T., and Vitart, F.: The new ECMWF seasonal forecast system (System 4), ECMWF, Reading, UK, ECMWF Technical Memorandum 656, 49 pp., 2011.

Mutai, C. C., Ward, M. N., and Coleman, A. W.: Towards the prediction of the East Africa short rains based on sea-surface temperature-atmosphere coupling, Int. J. Climatol., 18, 975-997, 1998.

Nicholson, S. E.: A review of climate dynamics and climate variability in Eastern Africa, in: The limnology, Climatology and Paleoclimatology of the East African Lakes, edited by: Johnson, T. C. and Odada, E. O., Overseas Publishers Association, Amsterdam, The Netherlands, 25-56, 1996.

Nicholson, S. E. and Nyenzi, B. S.: Temporal and spatial variability of SSTs in the tropical Atlantic and Indian Oceans, Meteorol. Atmos. Phys., 42, 1-17, 1990.

Ogallo, L. J.: Quasi-periodic patterns in the East Africa rainfall records, Kenya Journal of Science and Technology, A3, 43-54, 1982.

Ogallo, L. J., Janowiak, J. E., and Halpert, M. S.: Teleconnection between seasonal rainfall over East Africa and Global seas surface temperature anomalies, J. Meteorol. Soc. Jpn., 66, 807-822, 1988.

Ogallo, L. J., Bessemoulin, P., Ceron, J. P., Mason, S., and Connor, S. J.: Adapting to climate variability and change: The climate outlook forum process, WMO Bulletin, 57, 93-102, 2008.

Simmons, A. J.: Numerical prediction: Some results from operational forecasting at ECMWF, Adv. Geophys., 29, 305-338, 1986.

WMO (World Meteorological Organisation): Press release December 2009, WMO No. 872, 2009.

Yuan, X. and Wood, E. F.: Multimodel seasonal forecasting of global drought onset, Geophys. Res. Lett., 40, 4900-4905, doi:10.1002/grl.50949, 2013. 